\title{
Functional independence in the Finnish spinal cord injury population
}

\author{
Kirsi Majamäki ${ }^{1,14}$, Susanna Tallqvist $\mathbb{D}^{2,14}{ }^{凶}$, Aki Vainionpää ${ }^{3}$, Eerika Koskinen D $^{4}$, Anna-Maija Kauppila ${ }^{5}$, Paula Bergman (D) ${ }^{6}$, \\ Heidi Anttila ${ }^{7}$, Harri Hämäläinen ${ }^{8}$, Anni Täckman ${ }^{9}$, Mauri Kallinen ${ }^{10,11}$, Jari Arokoski ${ }^{8}$ and Sinikka Hiekkala ${ }^{12,13}$
}

(c) The Author(s) 2021

STUDY DESIGN: A cross-sectional survey of the Finnish population with spinal cord injury (FinSCI database).

OBJECTIVES: To describe the functional independence of the population with spinal cord injury (SCI) in Finland and to identify how generic and lesion characteristics affect their functional independence.

SETTING: The participants were recruited from the registers of three SCl outpatient clinics responsible for lifelong follow-up and care for people with $\mathrm{SCl}$ in Finland.

METHODS: The data were retrieved from FinSCI $(n=1772)$. The response rate was $50 \%(n=884)$. The Spinal Cord Independence Measure-Self Report (SCIM-SR) was used. The data were analyzed with univariate testing, factor analyses, and multiple linear regression models.

RESULTS: The median (percentiles 25; 75) SCIM-SR total score was 76.0 (58.8; 89.0), and the score was 18.0 (13.0; $20: 0)$ for the selfcare sub-scale, $33.0(25.0 ; 39.0)$ for the respiration and sphincter management sub-scale and $29.0(16.0 ; 36.8)$ for the mobility subscale. The higher the neurological level in groups AIS A, B, and C, the lower the functional ability. Group AIS D at any injury level had the highest level of functional ability. Age and the number of years since injury negatively influenced the SCIM-SR scores for every sub-scale.

CONCLUSION: Based on the International Spinal Cord Injury Core Data Set, the severity of SCl can differentiate persons with SCI according to their functional ability. The results suggest that $\mathrm{SCl}$ affects individuals' health more than ageing alone does, thereby reducing the functional ability and independence of persons with $\mathrm{SCl}$ over time.

Spinal Cord (2022) 60:628-634; https://doi.org/10.1038/s41393-021-00700-x

\section{INTRODUCTION}

Spinal cord injury (SCl) can cause sensory and motor loss and changes in the autonomic nervous system [1]. Tetraplegia, a cervical $\mathrm{SCl}$, affects the sensory and motor function of the arms, body, and legs. Individuals with lesions at the C4 or higher may need ventilation assistance. Paraplegia, a thoracic or lumbar $\mathrm{SCl}$, impacts the function of the trunk and legs [2]. Neurological status is the strongest predictor of functional independence [3]. Other factors such as secondary health conditions, psychological, social, and environmental supports, and cognitive ability can also influence the outcomes. For individuals with a complete $\mathrm{SCl}$, the optimal level of functional independence can be estimated using outcome-based practice guidelines. For persons with incomplete $\mathrm{SCl}$, the goal-setting process for the function is more individualised [4].
The International Standards for Neurological Classification of SCI (ISNCSCI) are used to assess the neurological level and completeness of $\mathrm{SCl}$. The International $\mathrm{SCl}$ Core Data Set provides recommendations for the standardisation of reporting, and it is recommended that the severity of $\mathrm{SCl}$ be grouped by ISNCSCI [5]. The purpose of the International SCl Core Data Set is to give SCl studies a standardised way of collecting and reporting data, which would enable the results of one $\mathrm{SCl}$ study to be compared with those of another [6].

As part of the Finnish Spinal Cord Injury Study (FinSCI) [7], this study aimed to describe the functional independence of a population with $\mathrm{SCl}$ in Finland by using the spinal cord independence measure-self report (SCIM-SR) [8]. It also examined how generic and lesion characteristics, classified by the recommendations of the International $\mathrm{SCl}$ core Data set, affected SCIM-SR scores [5].

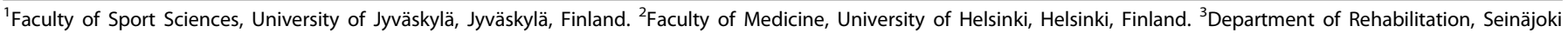

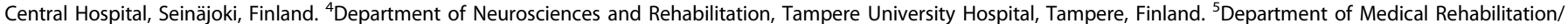

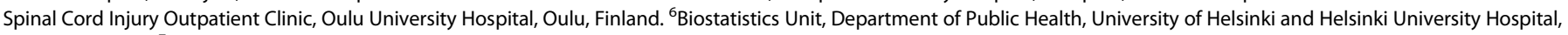

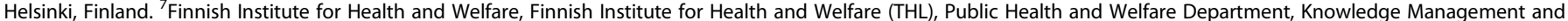

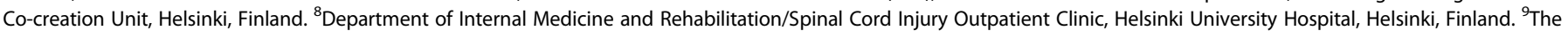

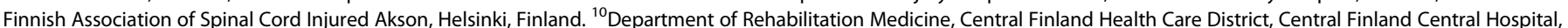

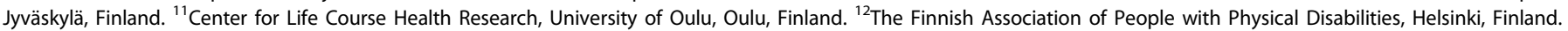
${ }^{13}$ Validia Rehabilitation, Helsinki, Finland. ${ }^{14}$ These authors contributed equally: Kirsi Majamäki, Susanna Tallqvist. ${ }^{凶}$ email: susannatallqvist@gmail.com
} 
Table 1. Eligible population of the FinSCl study divided into participants (N884) and non-respondents (N888).

\section{Participants N884 \\ n (\%)}

Gender

Female

Male

Age, years

\begin{tabular}{l}
$20-30$ \\
\hline $31-45$ \\
\hline $46-60$ \\
$61-75$ \\
$\geq 76$
\end{tabular}

Aetiology

Traumatic

Non-traumatic

Severity of $\mathrm{SCl}$

\section{C1-4 AIS A, B, and C}

C5-8 AIS $A, B$, and $C$

T1-S5 AIS A, B, and C

AIS $D$ at any injury level

Years since injury

$$
\begin{aligned}
& 1-5 \text { years } \\
& 6-10 \text { years } \\
& 11-15 \text { years } \\
& \geq 16 \text { years }
\end{aligned}
$$

307 (35\%)

577 (65\%)

(min 20, max 90, mean 61, SD 14)

median 63 IQR 53-71

34 (4\%)

108 (12\%)

238 (27\%)

386 (44\%)

118 (13\%)

492 (56\%)

392 (44\%)

95 (12\%)

$55(6 \%)$

$184(21 \%)$

550 (62\%)

$(\min 1, \max 67$, mean 11, SD 11)

median 7 IQR 4-14

$353(40 \%)$

227 (26\%)

$128(14 \%)$

$176(20 \%)$

\section{Non-respondents $\mathbf{N 8 8 8}$}

$n$ (\%)

$253(29 \%)$

$633(71 \%)$

(min 17, max 93, mean 54, SD 17)

median 55 IQR 40-68

96 (11\%)

$204(23 \%)$

$243(27 \%)$

$243(27 \%)$

$102(12 \%)$

527 (59\%)

361 (41\%)

$107(11 \%)$

$62(7 \%)$

209 (24\%)

510 (57\%)

(min 1, max 66, mean 10, SD 10)

0.52

median 6 IQR 4-14

$379(43 \%)$

$222(25 \%)$

111 (12\%)

$176(20 \%)$

\section{METHODS}

Design

FinSCl is a collaborative study among The Finnish Association of People with Physical Disabilities, The Finnish Association of Spinal Cord Injured Akson, The Finnish Institute of Health and Welfare, and the $\mathrm{SCl}$ outpatient clinics of three university hospitals (Oulu, Tampere and Helsinki). These three university hospitals are responsible for acute care, immediate rehabilitation and life-long multi-professional follow-up, care and rehabilitation of persons with $\mathrm{SCl}$ in all of Finland. The purpose of $\mathrm{FinSCl}$ is to identify factors related to the health and functioning of persons with $\mathrm{SCl}$, their challenges with accessibility, and how such factors are interconnected [7].

\section{Sample}

Study participants were recruited from the registers of the $\mathrm{SCl}$ outpatient clinics from the university hospitals in Oulu, Tampere and Helsinki. Their clinical data, consisting of general and lesion characteristics including ISNCSCI [5] to determine The American spinal injury association impairment scale classification (AIS) and the neurological level of the injury, were also collected from these registers. Inclusion criteria were as follows: age of at least 16 years; non-traumatic SCI (NTSCl) or traumatic SCI (TSCI); and AIS of $A, B, C$ or D. Patients with AIS E, living in an institute or with a congenital $\mathrm{SCl}$, progressive or neurodegenerative disease, multiple sclerosis, amyotrophic lateral sclerosis or Guillain-Barre syndrome were excluded. The detailed protocol, the precise patient selection process and the content of the formulated questionnaire have been presented elsewhere [7]. The questionnaire was sent to the participants in February 2019, and the answers were collected until the end of July 2019.

\section{Outcome measure}

This study evaluates the functioning of the Finnish population with $\mathrm{SCl}$ using the SCIM-SR [8], which was based on the clinician-administered SCIM III (spinal cord independence measure version III), an internationally used measure for the functional assessment of SCl populations [9]. The first version of SCIM was published in 1997 and was used as a multidisciplinary instrument to assess functioning in people with $\mathrm{SCl}$ [10].

SCIM-SR is a self-report outcome measure that is commonly used in community-based settings [11]. Self-reported indicators are easy to use and are targeted for a focus group [12]. Self-reports take less time [12, 13], require fewer resources [12] and are a cost-effective way of measuring functional ability [13]. They are valid for the evaluation of self-care functioning in populations with disabilities and can be used in inpatient and outpatient situations [12]. The results of SCIM-SR are correlated with those of the SCIM III [8, 14-16], which has good validity and reliability [8]. SCIM-SR has been used in a national study in Switzerland (SwiSCI) [17] and in the international survey $\mathrm{InSCl}$ [18]. The use of SCIM-SR has been recommended in outpatient and community settings, as well as in acute and post-acute rehabilitation settings [8]. It is useful for monitoring changes in functional independence [19]. SCIM-SR covers 12 of the 43 preselected categories from the International Classification of Functioning, Disability and Health (ICF) [20] from the FinSCl dataset [7].

SCIM-SR consists of 17 items divided into three sub-scales: self-care, respiration and sphincter management, and mobility. For each item, the person evaluates their present situation and their need for assistance to complete the activities. Self-care (score range 0-20) includes six items related to eating and drinking, washing, dressing and grooming. There were 2 items addressing upper body and lower body functions. Respiration and sphincter management (score range 0-40) were covered by four items, with questions about breathing, bladder and bowel management, and toilet use; three items addressed bladder and bowel management. There are nine items on mobility (score range 0-40), which address the need for assistance and the ability to move around. The SCIM-SR total score ranges between 0 and 100 . The higher the score, the better the individual's level of independent functioning [8].

\section{Statistical analyses}

Descriptive statistics were used to summarise the characteristics of the study population. The data were not normally distributed, so nonparametric tests were used. The SCl-related demographics are presented as 
means (and standard deviations); frequencies (and percentages); or medians (and $25 \%$ and $75 \%$ percentiles), depending on the distribution of the data. Factor analyses were used to explore SCIM-SR's ability to distinguish groups differing in $\mathrm{SCl}$ severity. Differences among the groups were examined with nonparametric tests (Kruskal-Wallis, Mann-Whitney $U$ test, Wilcoxon). The significance values for pairwise comparisons were adjusted by Bonferroni correction for multiple tests. In addition, multiple linear regression models were used to study how age, $\mathrm{SCl}$ severity and the number of years since injury are associated with the total score. $P$-values

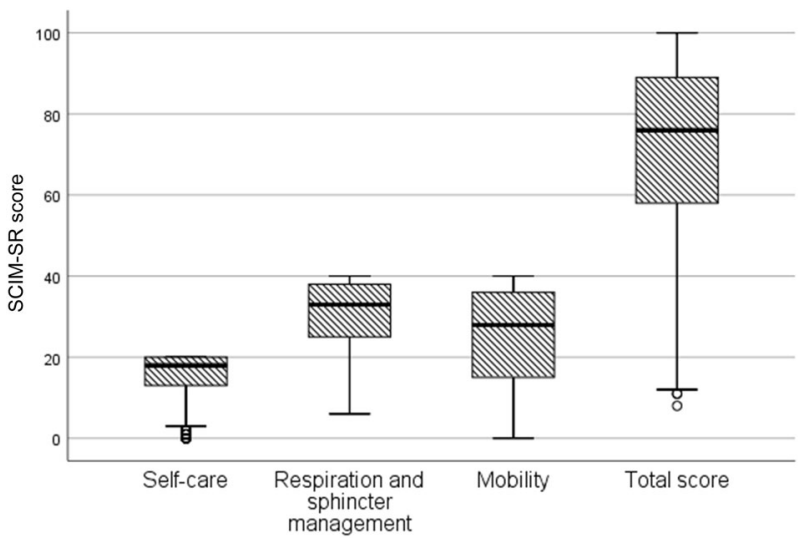

Fig. 1 The sub-scale and total scores of the SCIM-SR for the population with $\mathrm{SCl}$ in the FinSCl study $(n=884)$ (median, percentiles $25 ; 75$ ). Self-care sub-scale observed range $0-20$; respiration and sphincter management sub-scale observed range 6-40; mobility sub-scale observed range $0-40$; total score observed range $8-100$
$<0.05$ were considered statistically significant. Statistical analyses were carried out using SPSS version 24 software (SPSS Inc., Chicago, Illinois, USA). There were some missing values in the dataset (self-care $4.4 \%$, respiration and sphincter management $15.6 \%$, mobility $11.5 \%)$. The number of missing values did not differ between the $\mathrm{SCl}$ severity groups. The missing values were replaced with the medians for all sub-scales, and they were calculated separately for each $\mathrm{SCl}$ severity group.

\section{RESULTS}

\section{Characteristics of participants and non-respondents}

There was a statistically significant difference between the participants $(n=884)$ and the non-responders $(n=888)$ in gender and age (Table 1). Younger persons answered less frequently, and persons aged 61-75 and females participated actively.

The youngest participant was 20 years old, which is why the first age group is 20-30 years. The oldest participant was 90 years of age. Two participants used a ventilator and were included in groups C1-4AIS A, B and C based on the recommendations of the International Spinal Cord Injury Core Data Set [5]. There was one transgender participant who was grouped by gender according to the hospital records (Table 1). The shortest time since injury was 11 months, and this value was rounded up to one year. There were some statistically significant differences in the distributions of generic and lesion characteristics among the participants, which are presented in the Supplement (Supplementary Table A).

Regarding self-care, 292 (34\%) of the respondents received the maximum score; regarding respiration and sphincter management, $176(21 \%)$ received the maximum score; for mobility, 162 (18\%) received the maximum score; and 77 (10\%) received the maximum total score, respectively (Fig. 1).

Table 2. Crosstabulation of gender, age in years, the severity of SCl, aetiology, years since injury and SCIM-SR sub-scales self-care, respiration and sphincter management, mobility and total score in the Finnish spinal cord injury (FinSCl) survey.

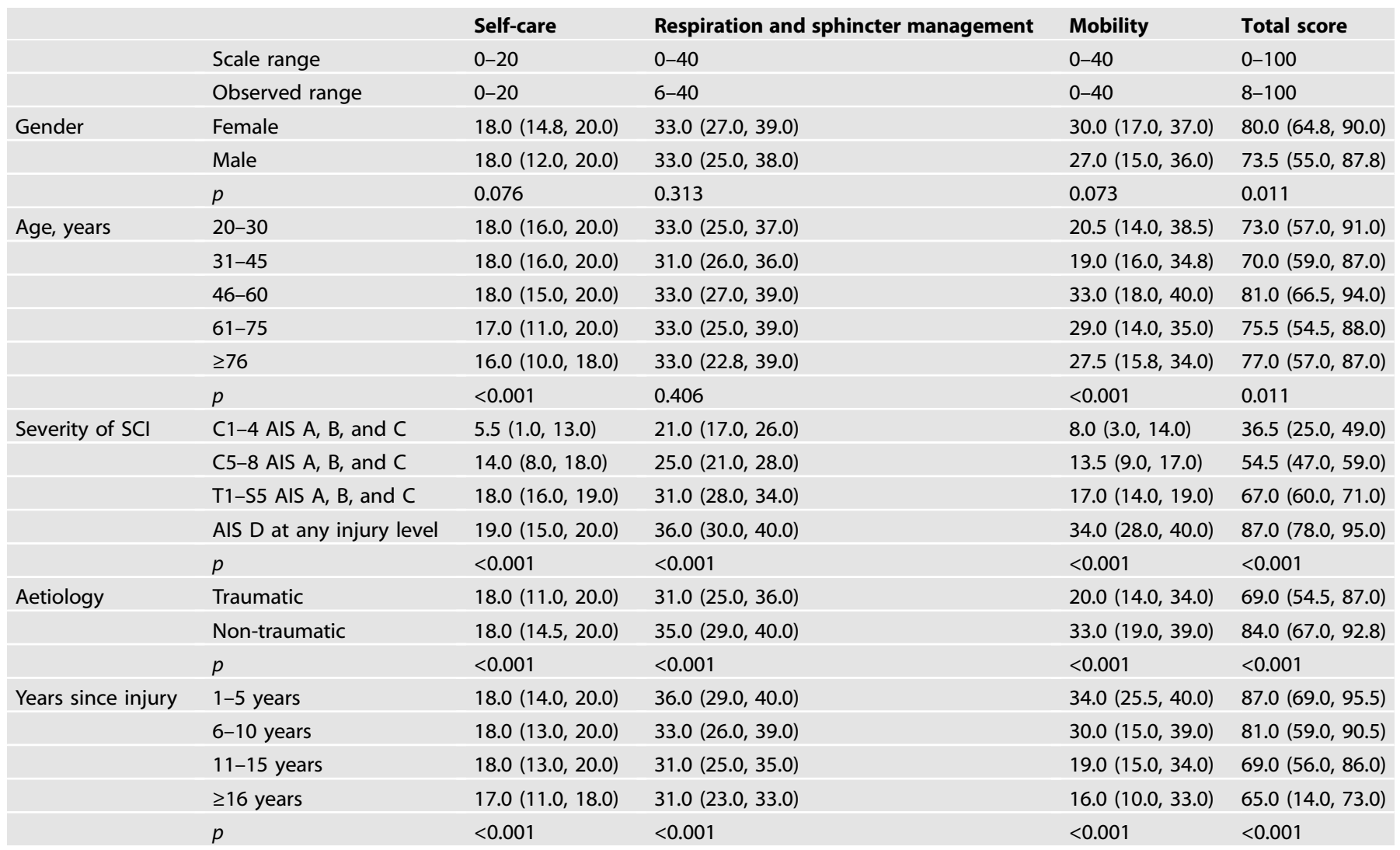

All scores are expressed as median with ( $25 \%$ and $75 \%$ percentiles). 


\section{Total score}

Gender, age, the severity of $\mathrm{SCl}$, aetiology and the number of years since injury had statistically significant effects on the SCIM-SR total score (Table 2). The females had higher scores than the males. The scores decreased with the number of years since injury, and there was a statistically significant difference between all the years since injury groups in the pairwise comparisons. The 46-60 age group had the highest score, and the 31-45 age group scored lowest. There was a statistically significant difference between the groups aged 31-45 and 46-60 and between those aged 46-60 and 61-75. AIS $D$ at any injury level (later referred to as group AIS D) scored highest, and group C1-4 AIS A, B and C had the lowest scores. In the pairwise comparisons of the $\mathrm{SCl}$ severity groups, there were significant differences between group AIS D and all the other $\mathrm{SCl}$ severity groups and between groups C1-4 AIS A, B and C and T1-S5 AIS A, B and C. The NTSCl group had higher scores than did the $\mathrm{TSCl}$ group.

The SCIM-SR scores can distinguish the $\mathrm{SCl}$ severity groups. AIS $D$ and T1-S5 AIS A, B and C groups were clearly distinguished from the other groups, but groups C1-4 AIS A, B and C and C5-8AIS A, B and $C$ seemed more difficult to separate from one another (Supplement; Supplementary statistical analyses, Supplementary Table B, and Supplementary Fig. A).

\section{Self-care sub-scale}

Ageing decreased individuals' ability to perform self-care, as shown by the pairwise comparisons between the groups aged 46-60 years and 61-75 years and between those 76 years or older and all other age groups (Table 2). The severity of $\mathrm{SCl}$ was a significant factor influencing functioning. Group AIS D had the highest scores, and group C1-4AIS A, B and C had the lowest. The scores for functioning were significantly different in the pairwise comparisons between all $\mathrm{SCl}$ severity groups. There was a statistically significant difference between the TSCI and NTSC groups, with the NTSCl group having higher scores. The persons who had been injured 5 or fewer years prior had the highest selfcare scores, and those who had been injured 16 or more years prior had the lowest self-care scores.

\section{Respiration and sphincter management sub-scale}

The respiration and sphincter management sub-scale scores differed statistically significantly according to all the lesion characteristics but not the generic characteristics (Table 2). Furthermore, in the pairwise analyses of the $\mathrm{SCl}$ severity groups, there was a statistically significant difference between all the groups except between group C1-4 AIS A, B and C and group C5-8AIS A, B and C. Group AIS D had the highest scores, and group C1-4 AIS A, B and C had the lowest. The TSCl group had statistically significantly lower scores than did the NTSCI group. The participants who had been injured 5 or fewer years ago had the highest scores, and those who had been injured 11 or more years ago had the lowest scores. In the pairwise analyses, the difference was statistically significant between all the years since injury groups.

\section{Mobility sub-scale}

The 31-45 age group had the lowest mobility scores, and the 46-60 age group had the highest (Table 2). In the pairwise comparisons of the age groups, there was a statistically significant difference between age groups 31-45 and 46-60, 46-60 and 61-75, and 46-60 and $\geq 76$. The severity of $\mathrm{SCl}$ was a notable factor influencing mobility. Group AIS D had the highest scores, and group C1-4 AIS A, B and C had the lowest scores. Group AIS D differed significantly from all other SCI severity groups, and in addition, there was a statistically significant difference between group C1-4 AIS A, B and C and group T1-S5 AIS $A, B$ and $C$. The NTSCl group had higher mobility scores than did the
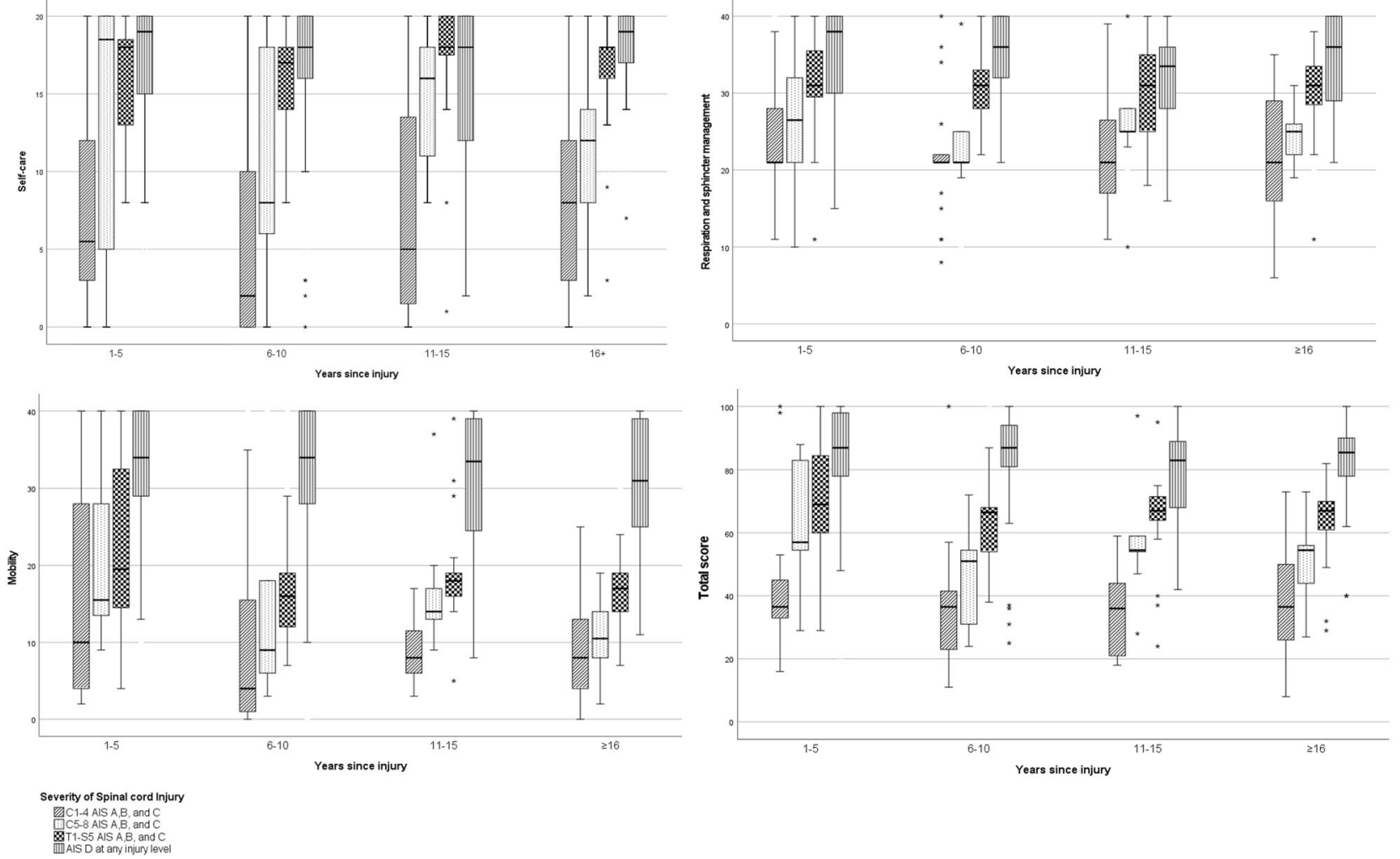

Fig. 2 Distribution of the severity of SCl across the years since injury groups in terms of the SCIM-SR sub-scales (self-care observed range $0-20$, respiration and sphincter management observed range 6-40, mobility observed range $0-40$ and total score observed range 8-100) (median, percentiles 25; 75). 
Table 3. Results of the linear regression analysis concerning the associations between SCIM-SR total scores and variables of age, severity of SCI groups (C1-4 A, B and C; C5-8 A, B and C; T1-S5 A, B and C; Group D) and years since injury groups (1-5; 6-10; 11-15; 216) in the FinSCl study.

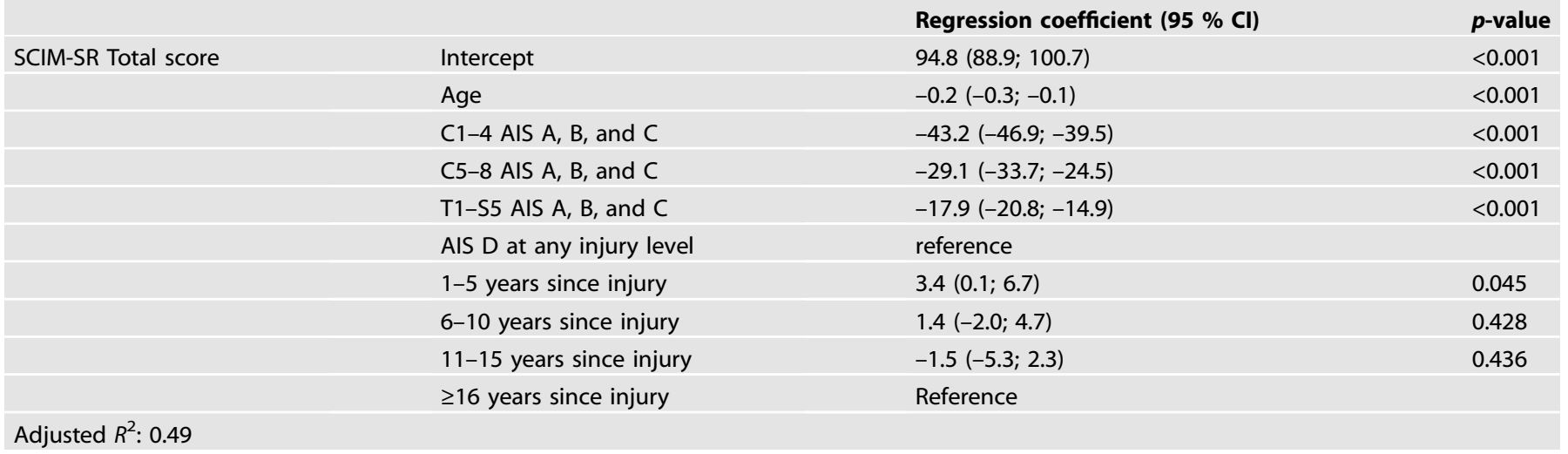

TSCl group. The participants who had been injured 5 or fewer years prior had the highest scores, and those injured 16 or more years prior had the lowest scores. The scores tended to decrease as the time since injury increased. As with the respiration and sphincter management sub-scale, there was a statistically significant difference between the years since injury groups.

\section{Interrelations of age and years since the injury with the SCI severity groups}

The effect of time since injury in years on the SCIM-SR scores was analysed separately within each $\mathrm{SCl}$ severity group (Fig. 2). For the self-care sub-scale, there was a statistically significant difference within group T1-S5 AIS A, B and C between the groups with 6-10 and $11-15$ years since the injury. For the respiration and sphincter management sub-scale, there were significant differences within group AIS D in the pairwise comparisons between groups 1-5 and 11-5 years since injury, and between the 6-10 and 11-15 years since injury groups. For the mobility sub-scale, there was a statistically significant difference within group C5-8 AIS A, B and C in the pairwise comparisons between the groups with 1-5 and 16 or more years since the injury. In addition, for the total scores of group AIS D, there was a statistically significant difference between the groups with 1-5 and 11-15 years since the injury. Despite some statistically significant differences, there was no consistency in the results (Fig. 2).

Multiple linear regression was run to understand the effects of age, the severity of $\mathrm{SCl}$ and the number of years since the injury on the SCIM-SR total score (Table 3). The levels of homoscedasticity and normality of the residuals were satisfactory, as assessed by visual inspection, and no significant outliers were present, as assessed by Cook's distance. The model was statistically significant $\mathrm{F}(7.802)=$ 111.52; $p<0.001$ and accounted for $49 \%$ of the variation in the SCIM-SR total score. Older age was associated with a lower SCIM-SR total score. Groups C1-4 AIS A, B and C, C5-8 AIS A, B and C, and T1-S5 AIS A, B and C were associated with lower SCIM-SR total scores than group AIS D. The 1-5 years since injury group had a higher SCIM-SR total score than did the $\geq 16$ years since injury group. The other years since injury groups did not differ statistically significantly from the $\geq 16$ years since injury group.

\section{DISCUSSION}

This study used SCIM-SR to assess functional independence among the Finnish population with $\mathrm{SCl}$. All generic and lesion characteristics had some impact on the scores, but the aetiology, the severity of $\mathrm{SCl}$ and the number of years since injury affected all sub-scores and total scores. Persons with NTSCI had a higher level of functional independence than persons with $\mathrm{TSCl}$. Those with the most severe $\mathrm{SCl}(\mathrm{C} 1-4$ AIS A, B and C) had the most limitations. In the group with ISNCSCI AIS A, B and C, the scores increased as the level of SCI decreased. Group AIS D had the highest level of functional ability. The time since injury negatively impacted the scores since the persons who were injured the earliest had the lowest level of functional independence. In addition, older persons tend to have lower total scores.

In the mobility sub-scale and total score, the two youngest age groups had the lowest scores. This contrast to our main results can be explained by the smaller group size and the higher incidence of $\mathrm{TSCl}$ in the youngest age groups. The missing values were replaced with the medians, and because of its nature, median imputation can introduce some bias into the results. This aspect should be noted when interpreting the results. The ceiling effect, that is, the high proportion of observations with a maximum score was considerable in the sub-scales of self-care and in respiration and sphincter management. This was mainly related to the overall good function of the participants, and to the large representative sample of persons with NTSCI in group AIS D.

Currently, two studies using SCIM-SR have been published $[19,21]$. Although the analysing methods of these studies differ from ours, Prodinger et al. [19] indicated that the generic and lesion characteristics are relevant factors in the analyses of SCIM$\mathrm{SR}$ results, and we agree with that. Based on the results of InSCI [21], persons with complete tetraplegia had more problems with their functional independence than persons with paraplegia or incomplete tetraplegia, as in our study. In contrast to our results, the time since injury did not have a similar kind of negative impact on functional independence; for example, dressing lower body and grooming (parts of the self-care sub-scale) and moving $<100 \mathrm{~m}$ (part of the mobility sub-scale) were more problematic among the participants who had been injured 5 or fewer years prior in comparison to those who have been injured for a longer time [21]. The fact that the Finnish population with $\mathrm{SCl}$ was older than the population in InSCl might partly explain this result.

Clinician-administered SCIM III has been used in several studies, providing valuable information on functional independence among the population with $\mathrm{SCl}$ [22-28]. Unfortunately, neither SCIM III nor the self-reported SCIM (SCIM-SR) based on SCIM III has thresholds or reference values, and they are not validated for persons with NTSCl. Therefore, a more specific evaluation of the level of functional independence in the Finnish population with $\mathrm{SCl}$ is not possible.

\section{Strengths and limitations}

One of the strengths of this study is that the participants, along with their generic and lesion characteristics, were collected from the registers of $\mathrm{SCl}$ outpatient clinics. This made it possible to find 
the majority of persons with $\mathrm{SCl}$ in Finland and combine their characteristics reliably. Persons with $\mathrm{SCl}$ actively participated in planning and performing the FinSCl study, which can be seen as an undisputed asset. The response rate was only 50\%; however, this is satisfying in comparison to response rates varying from $23 \%$ to $54 \%$ in countries with defined sampling frames in InSCI [29].

Since the clinical examinations of the participants were performed by several different doctors and physiotherapists between 2000 and 2018, we recognise that the validity and reliability of the ISNCSCI results in our study may be questioned. In addition, ISNCSCI is not fully validated to measure persons with NTSCl. However, the most recent data found in the medical records was used, and persons with progressive or neurodegenerative diseases were excluded.

Analyses of the participants and non-respondents indicate that younger individuals with $\mathrm{SCl}$ were not extremely interested in responding to the survey, which might have altered the overall division of the participants according to $\mathrm{SCl}$ severity. The high incidence of individuals in SCl severity group AIS D was expected since $\sim 65 \%$ of the Finnish $\mathrm{SCl}$ population belongs to this group [30]. Largely because of this factor, some of the data are skewed, and the results must be interpreted with caution. Group AIS D differed significantly from the other $\mathrm{SCl}$ severity groups since it included more elderly persons, persons with NTSCI and persons who were injured fewer than 5 years prior. Additionally, selfreporting can be seen as doubtful. Participation of persons with the most severe $\mathrm{SCl}$ might be affected by the need for assistance in answering. The evaluation of sphincter management was challenging and can reflect the higher number of non-responding participants to that specific area of questioning.

\section{CONCLUSIONS AND FUTURE DIRECTIONS}

Our results suggest that $\mathrm{SCl}$ consumes more health than ageing alone does. The time since injury had a negative impact on functional independence as early as 6-10 years after $\mathrm{SCl}$, and the scores decreased between all the time-since-injury groups. This result is worrying and seems to differ from the international results [21]. Recognising this problem can help us plan for future needs regarding health care and rehabilitation for persons with $\mathrm{SCl}$. In particular, the needs of elderly persons with $\mathrm{SCl}$ should be carefully evaluated and supported.

This study provided baseline information on the functional ability of the Finnish population with SCl. Although SCIM-SR does not involve psychosocial components that are relevant for everyday life, it evaluates a person's ability to manage their daily routines. These results can be used as reference values in the future. The FinSCI project itself promoted SCIM-SR for use in $\mathrm{SCl}$ outpatient clinics, and we believe that SCIM-SR will be widely used in Finland in the future.

The third conclusion of this study is that, although the ISNCSCI is not a functional measure, we think that grouping persons by $\mathrm{SCl}$ severity based on the ISNCSCl and the standardisation of reporting by the International SCl Core Data Set [5] are well suited for the SCIM-SR analyses. SCIM-SR should be further developed to account for these standardisations. In addition, when measuring persons with good functional independence (as group AIS D in our study), it would be desirable if SCIM-SR could describe the possible differences in functioning in even more detail and avoid the possible ceiling effect.

It would be useful if future research could determine internationally evaluated reference values for SCIM-SR. In addition, analyses of personal changes in functional ability that occur over time would provide the most useful information. This detailed information may help both persons with $\mathrm{SCl}$ and professionals working with them to estimate levels of functional independence and set realistic goals for rehabilitation to improve the daily lives of persons with $\mathrm{SCl}$.

\section{DATA AVAILABILITY}

The authors will consider any reasonable requests to access the data.

\section{REFERENCES}

1. Kirshblum SC, Burns SP, Biering-Sorensen F, Donovan W, Graves DE, Jha A, et al. International standards for neurological classification of spinal cord injury (revised 2011). J Spinal Cord Med. 2011;34:535-546.

2. Bickenbach J, Boldt I, Brinkhof M, Chamberlain J, Cripps R, Fitzharris M. A global picture of spinal cord injury. In: Bickenbach J, Officer A, Shakespeare T, von Groote $\mathrm{P}$, (eds.) International perspectives on spinal cord injury. Geneva: WHO; 2013. p. 13-41.

3. Middleton JW, Truman G, Geraghty TJ. Neurological level effect on the discharge functional status of spinal cord injured persons after rehabilitation. Arch Phys Med Rehabil. 1998;79:1428-1432.

4. Consortium for Spinal Cord Medicine. Outcomes following traumatic spinal cord injury: clinical practice guidelines for health-care professionals. Paralyzed Veterans of America; 1999.

5. Biering-Sorensen F, DeVivo MJ, Charlifue S, Chen Y, New PW, Noonan V, et al. International spinal cord injury core data set (version 2.0)-including standardization of reporting. Spinal Cord. 2017;55:759-764.

6. Devivo MJ. Epidemiology of traumatic spinal cord injury: trends and future implications. Spinal Cord. 2012;50:365-372.

7. Tallqvist $S$, Anttila $H$, Kallinen $M$, Koskinen $E$, Hämäläinen $H$, Kauppila A-M, et al. Health, functioning and accessibility among spinal cord injury population in Finland: protocol for the FinSCl study. J Rehabil Med. 2019;51:273-280.

8. Fekete C, Eriks-Hoogland I, Baumberger M, Catz A, Itzkovich $M$, Luthi $H$, et al. Development and validation of a self-report version of the spinal cord independence measure (SCIM III). Spinal Cord. 2013;51:40-47.

9. Itzkovich M, Shefler H, Front L, Gur-Pollack R, Elkayam K, Bluvshtein V, et al. SCIM III (spinal cord independence measure version III): reliability of assessment by interview and comparison with assessment by observation. Spinal Cord. 2018;56:46-51.

10. Catz A, Itzkovich M, Agranov E, Ring H, Tamir A, SCIM -. Spinal cord independence measure: a new disability scale for patients with spinal cord lesions. Spinal Cord. 1997;35:850-856.

11. Dorevitch $M$. The 'questionnaire' versus the 'direct observation' approach to functional assessment. Br J Rheumatol. 1988;27:326.

12. Hoenig $\mathrm{H}$, Hoff J, Mclntyre L, Branch LG. The self-reported functional measure: predictive validity for health care utilization in multiple sclerosis and spinal cord injury. Arch Phys Med Rehabil. 2001;82:613-618.

13. Hoenig H, Mclntyre L, Sloane R, Branch LG, Truncali A, Horner RD. The reliability of a self-reported measure of disease, impairment, and function in persons with spinal cord dysfunction. Arch Phys Med Rehabil. 1998;79:378-387.

14. Michailidou C, Marston L, De Souza LH. Translation into Greek and initial validity and reliability testing of a modified version of the SCIM III, in both English and Greek, for self-use. Disabil Rehabil. 2016;38:180-188.

15. Aguilar-Rodríguez M, Peña-Pachés L, Grao-Castellote C, Torralba-Collados F, Hervás-Marín D, Giner-Pascual M. Adaptation and validation of the Spanish selfreport version of the Spinal Cord Independence Measure (SCIM III). Spinal Cord. 2015;53:451-454.

16. Bonavita J, Torre M, China S, Bressi F, Bonatti E, Capirossi R, et al. Validation of the Italian version of the spinal cord independence measure (SCIM III) self-report. Spinal Cord. 2016;54:553-560.

17. Brinkhof MWG, Fekete C, Chamberlain JD, Post MWM, Gemperli A, SwiSCI Study Group. Swiss national community survey on functioning after spinal cord injury: protocol, characteristics of participants and determinants of non-response. J Rehabil Med. 2016;48:120-130.

18. Gross-Hemmi MH, Post MW, Ehrmann C, Fekete C, Hasnan N, Middleton JW, et al. Study protocol of the international spinal cord injury (InSCl) community survey. Am J Phys Med Rehabil. 2017;96:S23-S34.

19. Prodinger B, Ballert CS, Brinkhof MW, Tennant A, Post MW. Metric properties of the spinal cord independence measure-self report in a community survey. Rehabil Med. 2016;48:149-164.

20. WHO. International classification of functioning, disability and health (ICF). Geneva: World Health Organization; 2001.

21. Ehrmann C, Reinhardt JD, Joseph C, Hasnan N, Perrouin-Verbe B, Tederko $P$, et al. Describing functioning in people living with spinal cord injury across 22 countries: a graphical modeling approach. Arch Phys Med Rehabil. 2020:101:2112-2143.

22. Osterthun R, Tjalma TA, Spijkerman DCM, Faber WXM, van Asbeck FWA Adriaansen JJE, et al. Functional independence of persons with long-standing motor complete spinal cord injury in the Netherlands. J Spinal Cord Med. 2020:43:380-387. 
634

23. Jörgensen S, Iwarsson S, Lexell J. Secondary health conditions, activity limitations, and life satisfaction in older adults with long-term spinal cord injury. PMR. 2017;9:356-366.

24. Cowan RE, Anderson KD. Replication and novel analysis of age and sex effects on the neurologic and functional value of each spinal segment in the US healthcare setting. Spinal Cord. 2019;57:156-164.

25. Kaminski L, Cordemans V, Cernat E, M'Bra KI, Mac-Thiong JM. Functional outcome prediction after traumatic spinal cord injury based on acute clinical factors. J Neurotrauma. 2017;34:2027-2033.

26. Tomioka Y, Uemura O, Ishii R, Liu M. Using a logarithmic model to predict functional independence after spinal cord injury: a retrospective study. Spinal Cord. 2019:57:1048-1056.

27. Ariji $Y$, Hayashi $T$, Ideta $R$, Koga $R$, Murai $S$, Towatari $F$, et al. A prediction model of functional outcome at 6 months using clinical findings of a person with traumatic spinal cord injury at 1 month after injury. Spinal Cord. 2020;58:1158-1165.

28. Corallo V, Torre M, Ferrara G, Guerra F, Nicosia G, Romanelli E, et al. What do spinal cord injury patients think of their improvement? A study of the minimal clinically important difference of the spinal cord independence measure III. Eur J Phys Rehabil Med. 2017;53:508.

29. Fekete C, Brach M, Ehrmann C, Post MWM, InSCl, Stucki G. Cohort profile of the international spinal cord injury community survey implemented in 22 countries. Arch Phys Med Rehabil. 2020;101:2103-2111.

30. Koskinen $E$, Väärälä $E$, Alen $M$, Kallinen $M$, Vainionpää A. Selkäydinvammojen ilmaantuvuus on ennakoitua suurempi [Incidence of spinal cord injuries in Finland higher than expected. Lääkärilehti. 2017;39:2160-2165b.

\section{ACKNOWLEDGEMENTS}

We want to thank MSc and Ph.D. student Joonas Poutanen from the University of Helsinki/Validia Rehabilitation for all the comments and feedback. Funding:This study was supported by the Finnish Association of People with Physical Disabilities (pr42105, 2017-2020); Oulu University Hospital (grant VTR K86709, 20.10.2017); Department of Internal Medicine and Rehabilitation, Helsinki University Hospital (grants HUS/53/2017 §9, 9.6.2017, HUS/76/2018 §11, 18.4.2018 and HUS/174/201 §1, 12.4.2019); and Validia Rehabilitation (grant HUS-VTR 9.3.2017).

\section{AUTHOR CONTRIBUTIONS}

$\mathrm{SH}$ was the principal investigator and director of the whole FinSCI study. ST collected the clients' data from the registers of the SCl outpatient clinics. ST and SH formulated the questionnaire, and all authors and seven persons with $\mathrm{SCl}$ provided feedback on it. All authors helped design and provide feedback on the report. KM analysed the data. KM and ST interpreted the results and wrote the report.

\section{FUNDING}

Open access funding provided by University of Helsinki including Helsinki University Central Hospital.

\section{COMPETING INTERESTS}

The authors declare no competing interests.

\section{ETHICS STATEMENT}

The study was approved by the HUS Coordinating Ethics Committee (HUS/1776/2017).

\section{ADDITIONAL INFORMATION}

Supplementary information The online version contains supplementary material available at https://doi.org/10.1038/s41393-021-00700-x.

Correspondence and requests for materials should be addressed to Susanna Tallqvist.

Reprints and permission information is available at http://www.nature.com/ reprints

Publisher's note Springer Nature remains neutral with regard to jurisdictional claims in published maps and institutional affiliations.

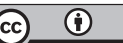

Open Access This article is licensed under a Creative Commons Attribution 4.0 International License, which permits use, sharing, adaptation, distribution and reproduction in any medium or format, as long as you give appropriate credit to the original author(s) and the source, provide a link to the Creative Commons license, and indicate if changes were made. The images or other third party material in this article are included in the article's Creative Commons license, unless indicated otherwise in a credit line to the material. If material is not included in the article's Creative Commons license and your intended use is not permitted by statutory regulation or exceeds the permitted use, you will need to obtain permission directly from the copyright holder. To view a copy of this license, visit http://creativecommons. org/licenses/by/4.0/.

(c) The Author(s) 2021 